\title{
BREVE HISTORIA DE LOS INICIOS DEL CINE GASTRONÓMICO: 1973-2010
}

\section{Cookery in contemporary cinema: $1973-2010$}

\author{
Rafael CARTAY; Exio Isaac CHAPARRO-MARTINEZ \\ Facultad de Ciencias Humanística y Sociales. Universidad Técnica de Manabí (Ecuador). \\ Autor para correspondencia: Exio Isaac Chaparro-Martinez \\ Correo electrónico: exiochaparro@gmail.com
}

Recibido: 21 de febrero de 2021

Aceptado: 21 de abril de 2021

\begin{abstract}
Resumen
En este artículo estudiamos el inicio del cine gastronómico como un género cinematográfico, considerando el período que va desde 1973, cuando apareció La Grande Bouffé (La Gran Comilona), el film considerado inaugural del género de cine gastronómico, hasta el año 2010 cuando, consolidado el género, se observó una gran rivalidad entre los productores y directores europeos y estadounidenses. Se efectuó una investigación documental para analizar las contribuciones que diversos investigadores han realizado sobre el tema. Esta revisión bibliográfica sintetiza los resultados de investigaciones y establece el estado del arte. En ese período (1973-2010) se produjo una fuerte competencia en la industria cinematográfica, que terminó con la imposición de la supremacía de la cinematografía estadounidense en la producción de películas del género. Se verificó la evolución del género, tanto en sus aspectos cuantitativos como cualitativos, al igual que los factores responsables que explican la hegemonía estadounidense.

Palabras clave: género cinematográfico; gastronomía; cine gastronómico; alimentación y cine.

\section{Abstract}

In this article we study the beginning of gastronomic cinema as a cinematographic genre, although it is the subject of controversy, considering a long period that covers almost four decades, since 1973, when La Grande Bouffé (The Great Feast) appeared, the film considered the inaugural
\end{abstract}


of the genre. gastronomic cinema, until 2010 when the genre was consolidated and a great rivalry was observed between European and American producers and directors. A documentary research was carried out to analyze the contributions that various researchers have made on the subject. This bibliographic review synthesizes the research results and establishes the state of the art. In that period (1973-2010) there was strong competition in the film industry, which ended with the imposition of the supremacy of American cinematography in the production of films of the genre. The evolution of the gender was verified, both in its quantitative and qualitative aspects, as well as the responsible factors that explain US hegemony.

Keywords: movie theater; gastronomy; gastronomic cinema; kitchen and cinema.

\section{Introducción}

«Cuando no se tiene nada qué comer es bueno, al menos, leer libros de cocina».

Honoré de Balzac.

"Cuando no se tiene nada qué comer es bueno,

al menos, ver películas de cocina». Cecil Bary.

Huberman ${ }^{1}$ en Los Bienes Terrenales del Hombre, se quejaba de la incongruencia de los libros de historia económica, que ponían a sus personajes a comer sin cancelar la cuenta. En la Edad Media los nobles vivían lujosamente y sin trabajar. Pero ¿quién pagaba la cuenta de tanto ocio y de tanto lujo? No se mencionaban los siervos de la gleba, esforzándose en los campos de cultivo del feudo, o al vasallo que servía en las tareas domésticas de las mansiones señoriales, como los responsables de que todo funcionara como en un cuento de hadas. Los personajes simplemente comían. Hablar de la alimentación era considerado una cosa ordinaria y de poca monta, de lo que no se hablaba en público porque lindaba en lo vulgar. La comida y los placeres de la mesa no trascendían del ámbito privado de las clases poderosas. La pompa de entonces la muestra el film Vatel (Roland Jaffé, 2000) sobre la vida de Francois Vatel, un cocinero del siglo XVII al servicio del príncipe de Condé. La Revolución Francesa (1789) desmontó el andamiaje feudal, lanzando al desempleo a los grandes cocineros de las mansiones señoriales, que se trasladaron a ciudades como París o Lyon, o se marcharon al exilio, para establecer los primeros restaurantes de lujo. La venta ambulante de comida era para los pobres, mientras que la comida en los grandes restaurantes era para los ricos. La comida cotidiana de la clase en ascenso se hacía en privado, en la intimidad del ámbito doméstico, resguardando la unidad familiar y las buenas costumbres. La otra comida, la extraordinaria, la celebratoria, llena de rituales y símbolos que mostraban el prestigio o condición social, que marcaba la identidad cultural y reforzaba la cohesión interna del grupo, se hacía algunas veces en público, en los grandes salones, para que tuviera un carácter de "didáctica» social. Cuando la comida celebratoria era en privado, se convertía en un derroche orgiástico en busca del placer, tal como hacían los nobles griegos y romanos en la Antigüedad, costumbre que luego imitaron los señores feudales.

La alimentación, presente desde el nacimiento hasta la muerte, e imprescindible para la sobrevivencia humana, ha sido considerada un hecho social total, que se manifiesta en gestos sociales organizados a partir de criterios culturales, económicos, nutricionales y simbólicos muy precisos. Entre los griegos y romanos, por ejemplo, la comida nutría el cuerpo y el alma. Desde Baco, el dios griego de la gastronomía, hasta Como, su contraparte latina, el acto de comer en público, o al menos en un ámbito más amplio que lo estrictamente doméstico, tenía una dimensión ritual, relacionada con el culto a los dioses, y una 
marcada connotación social y simbólica. Un análisis crítico de películas como La gran comilona, de Ferrari, no puede hacerse con propiedad si se desconocen algunos de esos aspectos, hasta el punto de que algunos críticos han tildado al film de Ferrari de "antigastronómico». La comida de celebración se convertía en un escenario superpuesto o en un espacio social alternativo para que la élite se expresara, tal como lo muestra Platón en El Banquete, o el latino Plauto en sus comedias del latino Plauto.

Eso no cambió en su esencia durante la Edad Media europea, sino sólo en la forma. Leopoldo Mozart, padre de W. A. Mozart, cuenta en una carta a su esposa, del 1 de febrero de 1764, «que el rey (se trataba de Luis XV) no come nunca en público, excepto el domingo por la noche en que se reúne toda la familia real para cenar». La mayor parte del tiempo, el rey comía solo, y la reina lo hacía aparte, con sus damas de compañía. Ese día, conocido como el "grand couvert», se escenificaba una especie de ceremonia. La corte y nobles invitados viajaban a Versalles para ver comer a la familia real. El momento culminante se producía cuando el rey exhibía su gran habilidad para descabezar un huevo duro con un solo golpe de cuchara. El ujier mayor anunciaba solemnemente: "Atención, el rey va a comer su huevo ${ }^{2}$. La comida era, entonces, y sigue siendo, como una puesta en escena, delimitando las categorías de comida privada y comida pública. De esa diferencia dio cuenta la expresión artística. Los griegos y romanos, en sus obras de teatro. Los pintores, en la Edad Media, en los bodegones o naturalezas muertas y con sus retratos de arquetipos sociales. Desde el fastuoso banquete hasta la humilde escena de un comedor de pan. Otros dejaron su huella en las incómodas salas de cocina, situadas fuera y luego dentro de la casa, y en las sucias chimeneas y en los caballetes de hierro que sostenían ollas sobre el fuego. Hasta que el fuego comenzó a ser domesticado en artefactos. Y los músicos incluyeron a la comida en sus canciones y óperas. Mozart compuso la música, y Lorenzo da Ponte el libreto, del drama Don Giovanni, de 1787: la única ópera conocida donde alguien canta con la boca llena de comida. Los literatos, por su parte, dejaron obras como Pantagruel (1532) y Gargantúa (1534), de Rabelais, o Don Quijote (1605-1615), de Cervantes, una obra con episodios como el de las bodas de Camacho.

Pero ¿qué pasó con el cine, y su relación con la alimentación humana, una preocupación constante de la gente ${ }^{3}$. El cine, en su rol mediático en la cultura de la modernidad, no podía ser indiferente ${ }^{4}$ y creó las condiciones para el nacimiento de un food film como subgénero ${ }^{5}$.

\section{Objetivos y metodología}

Esta investigación corresponde a una revisión documental sobre el inicio y la evolución del género de cine gastronómico, que gira en torno a la comida, para mostrar sus objetivos principales: la expresión e importancia de la actividad, y su regulación social durante el período 1973-2010.

\section{Resultados}

El cine se coló en el arte desde su nacimiento. El francés Louis Lumiére, el inventor del cinematógrafo, en 1895, realizó varias pruebas que se exhibieron ese ese año, en el Grand Café, lugar de encuentro de la intelectualidad parisina. Era la época de la "edad de oro» de la gastronomía parisina, en la que abundaban cafés y bistrós, en 1895 superaban los 2.000. Los restaurantes se habían multiplicado desde que, en 1756, Boulanger abrió en París su Champ d'Oiseaux, el primer restaurante francés con un cierto lujo ${ }^{6}$. El mismo año en que Lumière inventó el cinematógrafo, París tenía 927 restaurantes considerados superiores. Lumiere exhibió en aquel café, en 1895, dos breves muestras ligadas con el hecho alimentario. Una, El primer desayuno que registra a su hermano Auguste desayunando con su mujer y su 
hijo. La otra, De copas y amigos, que graba a sus amigos bebiendo en un bar. Tras ese inicio prometedor, el cine silenció su interés por la comida como acto social. Reduciéndolo a apariciones fugaces, como en The Big Shallow, de James Williamson, en 1901, en el que una persona amenaza con engullir la pantalla que lo filma ${ }^{7}$. 0 el Acorazado Potemkin, de Serge Eisenstein, en 1925, en el que la tripulación emplea el alimento descompuesto como medio de protesta. Debió esperarse hasta 1925 cuando Charles Chaplin, en el triple rol de escritor, director y actor principal, filmó La Quimera de Oro, que muestra un vagabundo, interpretado por Chaplin, engañando al hambre, con los cordones, el cuero y los clavos de una bota vieja, o haciendo de titiritero, simulando un baile con unas papas ensartadas con tenedores, a modo de piernas.

El tema culinario desapareció otra vez del cine. Se veían, de pasada, escenas donde la gente comía, pero descontextualizadas tanto de locación como de argumento. Como sucedió en las películas de Cecil B. Mille: Los Diez Mandamientos (1923), y Cleopatra (1934). Hasta que el hecho alimentario reapareció en el cine, con timidez, en Navidad en Connecticut (Peter Godfrey, 1945), con una columnista gastronómica, preparando una cena para los amigos de su jefe. Y luego, en 1973, con fuerza, al aparecer La gran comilona (Marco Ferreri, 1973), el film inaugural del género del cine gastronómico ${ }^{8}$. En cambio, para otros autores, como Parkhurst ${ }^{9}$, el cine gastronómico apareció luego, a mediados de la década de 1980. La estructura del nuevo género se levantó sobre cuatro pilares: el film de Ferreri, y tres más: ¿Quién mata a los grandes chefs de Europa?, de T. Kotcheff (1978); "El festín de Babette», de G. Axel, (1987), y «Como agua para chocolate», de A. Arau (1992). A partir de los 90, cada año apareció, al menos, una película gastronómica. Así se consolidó el género. Surgieron, además, paralelamente, desde los 90 , especialistas y espacios para el nuevo género. En España, el cinéfilo Pepe Barrena, creador del CineGourland en Getxo, Bibao, el primero de tres festivales especializados: el CineGourland en Bilbao; el Film \& Cook en Barcelona, y el «Festival de Cine Gastronómico» de la Universidad de La Laguna, en Canarias. Y secciones anexas a festivales de cine, como el de Huesca.

Entre 1973, con el estreno de La gran comilona de Ferreri, y 2010, que cierra con "18 comidas», de Coira, un lapso de 37 años, se produjeron 57 películas clasificables como cine gastronómico, a razón de 1,54 por año, que retrata la importancia del género, que estudiamos con el recurso de la «lista» de las obras ${ }^{10}$, presentadas en la tabla 1.

En síntesis, 57 films en 37 años, divisibles en dos subperíodos: el primero, entre 1973 y 2000, con 28 películas, para un promedio de 0,8 películas/año, y el segundo, entre 2001 y 2010, con 29 películas, para un promedio de 2,9 películas/año, triplicando al primero. El género cobró importancia desde 2001, con 2000 como punto de quiebre. Ese año se produjeron 6 películas, con Francia como líder. De las 28 películas del lapso 1973-2000, 10 (35,7 \%) fueron francesas. Pero desde 2001 aumentó la participación de EE. UU., con 22 de las 57 películas, o sea, 38,6\%. Esa importancia estadounidense se elevó en el lapso 2011-2010, con 14 (48,3\%) de las 29 películas ${ }^{10}$.

\subsection{El misterio de las clasificaciones}

El análisis de la supremacía de un cine sobre el otro depende de los criterios usados en la caracterización. Sin embargo, desconocemos la existencia de normas claras para catalogar a una película dentro de un género, bien sea cine gastronómico u Hospital films, un género por construir $^{11}$, o de un criterio preciso para definir un género. Por eso esta investigación opera en el terreno de las conjeturas. El género resulta una estrategia útil para agrupar películas que compartan ciertas características básicas ${ }^{12,13}$. Para otros autores $^{14}$ es un recurso cliché, que simplifica y

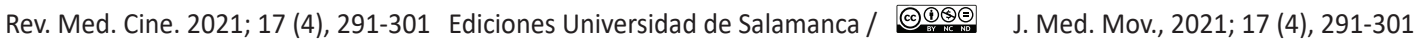
[ 294 ] 
BREVE HISTORIA DE LOS INICIOS DEL CINE GASTRONÓMICO: 1973-2010

RAFAEL CARTAY; EXIO ISAAC CHAPARRO-MARTINEZ

Tabla 1. Lista de las 57 películas del género gastronómico del período 1973-2010.

\begin{tabular}{|c|c|c|c|}
\hline Año & Título & Director & País \\
\hline 1973 & La gran comilona & Marco Ferreri & Francia-Italia \\
\hline 1976 & El ala o el anca & Claude Zizi & Francia \\
\hline 1978 & ¿Quién mata los chefs de Europa & Ted Kotcheff & Gran Bretaña-Alemania \\
\hline 1985 & Tampopo & Yuzo Itami & Japón \\
\hline 1986 & La mitad del cielo & Manuel Gutiérrez & España \\
\hline \multirow{2}{*}{1987} & El festín de Babette & Gabriel Axel & Francia \\
\hline & Baby Boom & Charles Shyer & EE.UU. \\
\hline 1988 & Mystic Pizza & Donald Petrie & EE.UU. \\
\hline 1989. & $\begin{array}{l}\text { El cocinero, el ladrón, su mujer y su } \\
\text { amante }\end{array}$ & Peter Greenaway & Gran Bretaña \\
\hline \multirow{2}{*}{1991} & Tomates verdes fritos & John Avnet & EE. UU. \\
\hline & Delicatessen & Jean-Pierre Jelwet & Francia \\
\hline 1992 & Como agua para chocolate & Alfonzo Arau & México \\
\hline \multirow{2}{*}{1993} & El olor de la papaya verde & Tran Anh Hung & Francia-Vietnam \\
\hline & Wallace \& Gromit & Nick Park & EE.UU. \\
\hline 1994 & Comer, beber y amar & Ang Lee & Francia- Taiwan \\
\hline \multirow{3}{*}{1995} & The Chinese feast & Tsui Hark, & Hong Kong \\
\hline & 301302 & Cheol-su Park & China \\
\hline & Al pequeño Marguery & Laurent Benegui & Francia \\
\hline \multirow{2}{*}{1996} & Una gran noche & Campbell Scott-Stanley Tucci & EE.UU. \\
\hline & Good of Cookery & Stephen Chow y Lik-Chi Lee & Hong Kong \\
\hline 1997 & El chef enamorado & Nana Dzhordzhadze & Bélgica-Francia \\
\hline \multirow{2}{*}{1998} & La cena & Ettore Scola & Italia \\
\hline & American cuisine & Jean-Yves Pitou & Francia \\
\hline \multirow{6}{*}{2000} & Hotel Splendide & Terence Gross & Gran Bretaña \\
\hline & Chocolat & Lasse Hallstrom & EE. UU. \\
\hline & Diner rush & Bob Giraldi & EE.UU. \\
\hline & Woman on top & Fina Torres & EE.UU. \\
\hline & Merci pour le chocolat & Claude Chabrol & Francia \\
\hline & Vatel & Roland Jaffé & Francia \\
\hline 2001 & Deliciosa Martha & Sandra Nettelbeck & Alemania \\
\hline 2002 & Jet lag & Daniele Thompson & EE.UU. \\
\hline \multirow{2}{*}{2003} & Un toque de canela & Tassos Boulmetis & Turquía \\
\hline & Kitchen stories & Bent Hamer & EE.UU. \\
\hline
\end{tabular}

Rev. Med. Cine. 2021; 17 (4), 291-301 Ediciones Universidad de Salamanca / @®@@ J. Med. Mov., 2021; 17 (4), $291-301$ [ 295 ] 
BREVE HISTORIA DE LOS INICIOS DEL CINE GASTRONÓMICO: 1973-2010

RAFAEL CARTAY; EXIO ISAAC CHAPARRO-MARTINEZ

\begin{tabular}{|c|c|c|c|}
\hline Año & Título & Director & País \\
\hline \multirow{3}{*}{2004} & Entre copas & Alexander Payne & EE.UU. \\
\hline & Super size me & Morgan Sporlock & EE.UU. \\
\hline & Mondovino & Jonathan Nossiter & EE.UU-Francia \\
\hline 2005 & Charlie y la fábrica de chocolate & Tim Burton & Gran Bretaña- EE.UU. \\
\hline 2006 & Un buen año & Ridley Scott & Gran Bretaña \\
\hline \multirow{3}{*}{2007} & Ratatouille & Brad Bird- Pixar & EE.UU. \\
\hline & No reservations & Scott Hicks & EE. UU. \\
\hline & La camarera & Adrienne Shelly & EE.UU. \\
\hline \multirow{3}{*}{2008} & Fuera de carta & Nacho Velilla & España \\
\hline & Gracias por el chocolate & Ron Howard & EE.UU. \\
\hline & Estómago & Marcos Jorge & Brasil \\
\hline \multirow{6}{*}{2009} & Soul kitchen & Fatih Akin & Alemania \\
\hline & Julie \& Julia & Nora Ephron & EE.UU. \\
\hline & Bottle shock & Randal Miller & EE.UU. \\
\hline & Dieta mediterránea & Joaquín Oristrell & España \\
\hline & Lluvia de albóndigas & Chris Miller-Phil Lord & EE.UU. \\
\hline & Mamouth & Lukas Moodysson & EE.UU. \\
\hline \multirow{6}{*}{2010} & Come, reza y ama & Ryan Murphy & EE.UU. \\
\hline & Bon appètit & David Pinillos & España \\
\hline & Yo soy el amor & Luca Guadagnino & Italia \\
\hline & Kofte & Michiel van Jaarsveld & Holanda \\
\hline & 18 comidas & Jorge Coira & España \\
\hline & Toast & S.J. Clarkson & Reino Unido \\
\hline
\end{tabular}

agrupa los estilos de una producción cultural. Para agrupar películas "gastronómicas» en torno a criterios unificadores, recurrimos al género de la literatura policial, policíaca o de crimen. Una novela o película es "policial» o "policíaca», cuando concurren varios elementos: un criminal o victimario, una víctima, un objeto homicida (arma blanca o de fuego, veneno, soga de ahorcamiento, objeto contundente, etc.), un móvil y el lugar de un crimen. Y, por supuesto, la presencia del policía o detective encargado de develar el enigma del asesinato. Cordero-Capio ${ }^{15}$ dice que debe existir un crimen enigmático y un proceso de investigación que busca aclararlo. En la novela policial hay que tener en cuenta que el delito es el tema básico, del cual se derivan todas las acciones, dramas y conflictos humanos ${ }^{16}$. La situación se complica cuando se produce una muerte, porque ésta, como hecho social, al igual que la alimentación, está cargada de simbolizaciones.

Apliquemos esos elementos para considerar a una película como gastronómica. Deben estar presente la mayoría de los siguientes elementos: un cocinero, un comensal, los ingredientes,

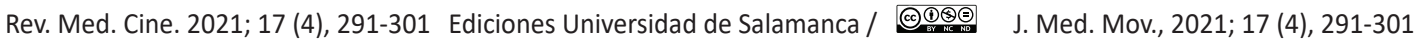


utensilios, artefactos y métodos de conservación y cocción, la preparación culinaria, los espacios donde se produce o consume una comida, las prácticas culturales relacionadas y los símbolos alimentarios asociados. Es conveniente, además, agregar la presencia de una suerte del crítico y la crítica gastronómica, que juzgan el resultado culinario. Y, por supuesto, una trama argumental que muestre episodios de interacción social entre los personajes, mediada por la comida.

El hecho alimentario, al igual que la salud o la muerte, está cargado de significaciones simbólicas. Cuando comemos, comemos alimentos y símbolos, porque los alimentos portan mensajes explícitos o implícitos insertos dentro de una cultura, y constituyen una manera de imaginarse el mundo y de insertarse en é $1^{17,18,19}$. Además, muchas sustancias son nutritivas y saludables, pero las hay también tóxicas, y algunas letales. $Y$ las sustancias son comestibles, biológica o culturalmente. Las películas del género tocan muchas aristas de la naturaleza y de la cultura, y obedecen a diversas motivaciones. Algunas plantean la lucha contra el hambre y los triunfos de la ciencia y la tecnología contra las carencias (Mamouth). Otras muestran los comportamientos ligados a conductas alimentarias individuales (Deliciosa Martha). Unas se refieren al perfeccionamiento de la alimentación como acto social y al nacimiento de una sensibilidad sensorial gastronómica (Soul Kitchen). Otras hablan de los excesos en la búsqueda del placer vinculado con la comida (La gran comilona, Dinner rush). Unas muestran al poder evocador de la comida y de la memoria gustativa (Un toque de canela). Otras se refieren al alimento y a la comida como elementos marcadores de identidad: estimulando la cohesión grupal y facilitando la diferenciación social (The chinese feast). Algunas plantean rivalidades entre agentes culinarios, empleando la cocina como escenario para mostrar los sentimientos humanos (No reservations, Chocolate, Como agua para chocolate). Para otras la comida es una instancia mediadora de lo político a través de signos de prestigio social y económico, reflejados en el lenguaje, las maneras en la mesa o la elección alimentaria (Vatel).

Hidalgo-Mari et al. ${ }^{20}$ simplifican esa amplia variedad de "motivos» en tres categorías: 1 ) La comida como reflejo de la diferencia social ( $L a$ Gran Comilona, El festín de Babette, El olor de la papaya verde); 2) La comida como exaltación de los sentidos (Como agua para chocolate, Chocolat, La camarera) y, por último, la comida como expresión representativa de la profesionalidad gastronómica (Julie \& Julia, Ratatouille, Baby Boom, Mystic Pizza, Deliciosa Martha). Dos Ramos ${ }^{21}$ clasifica las películas gastronómicas en tres categorías: 1) El alimento como excusa argumental, 2) el alimento como metáfora cultural, y 3) el alimento como ingrediente mágico, considerando a la cocinera como una hechicera. Hay que advertir que Dos Ramos ubica a los últimos films como Chick flicks: comedias poco serias.

Esas categorías, a pesar de su utilidad, parecen ser rígidas. Una película gastronómica es, por lo general, polisémica: cargada con muchos significados, por la propia naturaleza simbólica del acto alimentario. Barthes ${ }^{22}$ decía que la polisemia de la alimentación hacía que todas las situaciones humanas fueran atravesadas por ella. La cultura alimentaria es, en efecto, un sistema de creencias, representaciones, conocimientos y prácticas heredadas, aprendidas y ejercidas dentro de una cultura $^{18,23}$. La alimentación actúa como un sistema de símbolos que expresa las estructuras inconscientes de cada comunidad o grupo, y funciona como un referente para otorgar sentido a la existencia humana ${ }^{24}$. El cine ha resultado ser un excelente vehículo para expresar esa estrecha relación existente entre la alimentación y el sexo, dos móviles esenciales de la conducta humana, necesarios para entender la sobrevivencia del individuo y de la especie.

El cine actúa así, como un instrumento para expresar la cotidianeidad e interpretar la

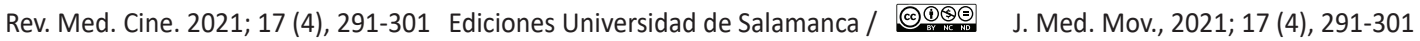
[ 297 ] 
alimentación a través de modelos culturales y sociales estructurados a manera de lenguajes. Lo dijo el arcipreste de Hita Juan Ruiz (1967: 101, estrofa 71), en el Libro de Buen Amor, del siglo XIV: «Como dice Aristóteles, cosa es verdadera, / el mundo por dos cosas trabaja: la primera / por haber mantenencia; la otra cosa era / por haber juntamiento con fembra placentera». Algunas películas, sin pertenecer al género, tocan tangencialmente el tema alimentario, de una manera magistral que nos impresionaron algunas escenas. Por ejemplo, el gazpacho de Mujeres al borde de una crisis de nervios, de Pedro Almódovar (1988), o el pan enmantequillado de Diva, de Jean-Jacques Beinex (1980) o los espaguetis de La Garçonniere, de Billy Wilder (1960). Otro film memorable fue $\mathrm{El}$ discreto encanto de la burguesía, de Luis Buñuel (1972), donde ocurren situaciones que impiden una gran cena, privando a esa clase de los estímulos de la ganancia y el placer de la comida.

Algunas películas se convirtieron en emblemáticas del género en la etapa inicial, entre 1973 y 1992. Por ejemplo, en ¿Quién mata...?, Korcheff realiza una inteligente crítica a la tendencia francesa de la Nouvelle Cuisine, promovida por el chef Paul Bocuse y los críticos gastronómicos Gault y Millau, que propusieron, en los 90, el aligeramiento de los menús y el retorno a lo natural como un planteamiento saludable y dietético. El festín de Babette presenta la historia de Babette, una renombrada cocinera parisina de la década de 1870 que, por una desilusión amorosa, se refugió en una apartada aldea danesa. Años después prepara una comida memorable que es comparada por un visitante con la que elaboraba una famosa chef parisina llamada Babette. Este film recrea la memoria gustativa individual. En Tomates verdes fritos se plantea la posibilidad de regenerar la vida de una joven deprimida, abandonada y con sobrepeso, empleando como mediación una aleccionadora historia contada por una anciana. Como agua para chocolate muestra la historia de una mujer que pospone su amor, obligada por rígidas costumbres familiares, y sobrelleva su dolor refugiándose en el quehacer culinario.

\subsection{Un modelo del género}

Ratatouille, un film estadounidense de dibujos animados, es un buen modelo del género de cine en cuanto a su estructura, reconocida con el Oscar de la Academia de Cine. Si usted omite a la rata Remy, conectora con el público infantil, se encuentra con una película que llena los requisitos de un film clasificable como gastronómico. Se desarrolla en un ambiente idóneo: la cocina de un restaurante; un concepto y lema: "Cualquiera puede cocinar», y una tradición gastronómica representada por el fallecido chef Gusteau; las rivalidades entre los agentes culinarios (Alfredo Linguine y Skinner, y en otro plano, Alfredo y Colette); una preparación emblemática de la cocina regional francesa, que da nombre a la película, la ratatouille, que juega con la composición denominativa en la que aparece la palabra «rata»; una relación amorosa que comienza con un rechazo y una rivalidad inicial, y que termina en una desbordada pasión (Alfredo y Colette); la búsqueda del plato estrella (la sopa que debe reproducir Alfredo bajo la presión de Skinner); los cambios de ramo dentro del negocio de restauración y la búsqueda de la rentabilidad (la amenaza de convertir al bistró en un expendio de comida precocida); el problema de las sucesiones en un negocio familiar de restauración (Gusteau-Alfredo); la formación del cocinero (las lecciones de Remy a Alfredo); y, por último, la presencia intimidante de Anton Ego, un juez subjetivo, pero implacable en la apreciación especializada del producto culinario, como sucede en las clasificaciones de la Guía Michelín, creada en 1900. Los nombres de la película (Ratatouille, plato guisado de hortalizas de la cocina regional francesa, en particular de Niza) y de los personajes no fueron escogidos al azar: Gusteau (de gusto y agua, en

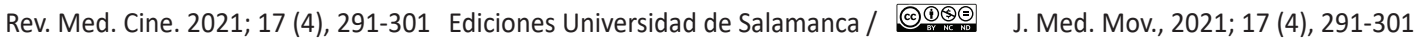
[ 298 ] 
francés), Remy (Rémy Martin, denominación de un famoso brandy o cognac de la región francesa de Cognac, y Rémy Pascal, el célebre inspector de la guía Michelin, que criticó la subjetividad del instrumento); Skinner (sicólogo estadounidense estudioso del aprendizaje y del condicionamiento operante), Alfredo Linguine (Alfredo, una salsa para pasta, y linguini, un formato de pasta italiana, de la región de la Campania), Colette (nombre femenino común en París y homónimo de una famosa novelista francesa), Ego (El Yo, el crítico narcisista, ególatra, que sólo considera su punto de vista y lo impone a los demás en un ámbito donde reina la subjetividad, y donde se otorga distinciones que exaltan o destruyen trayectorias); Pascal Rémy (el inspector de la guía Michelin que escribió un libro, El inspector se sienta a la mesa, en el que revela secretos del arbitraje, que puso en entredicho la pretendida «objetividad» de la crítica gastronómica mundial.

\subsection{A manera de conclusión}

Al revisar la lista de las 57 películas calificables dentro del género de cine gastronómico, concluimos que Estados Unidos es un second comer en el género que se convirtió, luego, en el más influyente, por encima de los first comers, como Francia. Nos preguntamos, entonces, ¿a qué se debe esa supremacía, tomando en cuenta que la gastronomía francesa es más reconocida que la estadounidense? Creemos hay dos razones que, sustentan esa supremacía. Una se relaciona con las características de la industria cinematográfica estadounidense. La otra se vincula con las características de la cocina y la gastronomía de Estados Unidos.

La industria estadounidense del cine es poderosa en virtud de sus elevados presupuestos y por disponer de un selecto plantel de directores, libretistas, productores y actores. Una industria sustentada por un enorme aparato publicitario y de comercialización de sus productos. Una industria competitiva, atenta a las tendencias del mercado. Una de las cuales apunta a la fusión entre cine y gastronomía, con el telón de fondo de las pasiones humanas. El poeta ruso Joseph Brodsky, Nobel de literatura en 1987, contó, en 2000, que parte de su vida la pasó en Leningrado, con el único contacto exterior de la radio y las películas de Hollywood. Para él, Tarzán contribuyó más a la desestalinización que todos los discursos políticos de Kruschov.

La otra razón se relaciona con las características de la cocina estadounidense, que no cuenta con una gran cocina. El poeta mexicano Octavio Paz, premio Nobel de literatura, señaló que: «La cocina norteamericana tradicional es una cocina sin misterios, alimentos simples, nutritivos y poco condimentados» (1979). Para él, la cocina estadounidense es modesta e insulsa, en especial si se compara con la maravillosa cocina mexicana, Esa escasa relevancia de la cocina tradicional de EE.UU. es el resultado del desinterés que mostraron los padres fundadores por su población originaria indígena y la de los otros, grupos inmigrantes: los negros africanos esclavizados, los latinoamericanos, los irlandeses, los asiáticos, los mexicanos despojados de su territorio. En América Latina los grupos inmigratorios formaron una singular y variada del mestizaje $(M)=$ $A+B+C+D$.

En Estados Unidos, en cambio, las culturas de los grupos de inmigrantes formaron un mestizaje inconcluso, donde $\mathrm{M}$ no es igual a la suma de $\mathrm{A}+$ $B+C+D$, en la que esos grupos continuaron separados, en barrios étnicos con su propia cocina. Una cocina fragmentada, que no logró integrar armoniosamente sus partes, que terminaron conformando un patchwork culinario, caracterizada por lo inauténtico de la mezcla, propia de la poca sensualidad heredada del protestantismo. Estados Unidos recibió solo entre 1820 y 1920 a 36 millones de inmigrantes. Braude ${ }^{25}$ enseña que un inmigrante viaja con dos maletas: una con sus enseres personales, su patrimonio material, y otra, con pedazos de cultura que le dan sentido a su vida. En esa maleta 
inmaterial viajan sus sueños, sus amores y su memoria gustativa. Adonde uno vaya, uno termina por reproducir su cultura, y en especial su cocina, que es su principal marca identitaria.

De allí que lo que uno llama cocina estadounidense no es más que un amplio espectro de cocinas de grupos de inmigrantes, que EE. UU. hizo suyo, apropiándoselo y recreándolo, para luego comercializarlo como propio, en el proceso de globalización de la cultura, y del paladar ${ }^{26}$. Son los Harlem, los Chinatown, Las Little Italy, las Pequeña Habana, o las grandes concentraciones de mexicanos, centroamericanos, irlandeses, hindúes, japoneses, que crearon, por su cuenta, barrios étnicos en el vientre de los grandes centros urbanos. Son las preparaciones culinarias que, de manera forzada, fueron reinventadas y apropiadas, como sucedió con el chop suey, el sushi-roll californiano, el sándwich inglés, la hamburguesa y el hot dog alemanes, el pretzel judío, el chili con carne, la fajita o el burrito tex-mex, la pizza y el macaroni \& cheese a la moda Nueva York, la doughnut o dona, la sopa de cebolla francesa, el croissant austríaco, la apple pie y muchos otros platos de inmigrantes. $\mathrm{O}$ de bebidas reconvertidas, como la cerveza alemana, aligerada en su contenido alcohólico, o el whisky bourbon hecho de maíz, o la Coca-Cola, la inteligente fusión de dos estimulantes, la coca sudamericana y la cola africana. La gente de la gran nación debía comer. Y lo hizo, en abundancia, apropiándose de los platos emblemáticos de sus inmigrantes, sin unirlos, para hacer una síntesis de esas notables cocinas tradicionales. Son las historias de cocina que nos cuenta la poderosa industria cinematográfica.

\section{Referencias}

1. Huberman L. Los bienes terrenales del hombre. Barcelona: Iguazú;1962.

2. Barría Aguiló J. Historias curiosas de la música y la gastronomía. Cenando con Mozart. Barcelona: Ediciones Robinbook; 2006.
3. Fernández-Armesto F. Historia de la comida: alimentos, cocina y civilización. Barcelona: Tusquets; 2004.

4. Lipovetsky G, Serroy J. La pantalla global. Cultura mediática y cine en la era hipermoderna. Barcelona: Anagrama. 2009.

5. Keller J.R. Food, film, and culture. A genre study. North California: Jefferson; 2006.

6. Neirinck E, Poulain JP. Historia de la cocina y de los cocineros. Barcelona: Editorial Zendrera Zariquiey; 2001.

7. Febrer-Fernández N. El estudio de la alimentación a través del análisis de textos fílmicos. Historia y Comunicación Social. 2014. 19.

8. Mérida P, García H. Cine a la carta. Barcelona: Ediciones Raima; 2009.

9. Parkhurst P. In Accounting for Taste: The Triumph of French Cuisine. Chicago: University of Chicago Press; 2004.

10. Santana-González JJ. Cine y gastronomía: análisis de textos fílmicos desde una perspectiva cultural. Trabajo de maestría. Granada: Universidad de Granada; 2017.

11. Levin LG, De Filippo D. Films and Science: quantification and analysis of the use of Science Fiction films in scientific papers. JCOM 2014; 13 (03):1-21.

12. Schatz T. Hollywood Genres. Formula, film making and the Studio System. New York: Random House; 1981.

13. Altman R. Los géneros cinematográficos. Barcelona: Paidós; 1999.

14. Hidalgo-Mari T, Segarra-Saavedra J, Rodríguez-Monteagudo E. El boom gastronómico en la gran pantalla. ¿Estamos ante un nuevo género cinematográfico? Fotocinema [Internet]. 2016 (13): 229-248.

15. Cordero-Carpio G. La novela policial en Ecuador. Tesis para la obtención del título de Magister en Estudios de la Cultura. Quito: Universidad Andina Simón Bolívar; 2013.

16. Galán-Herrera JJ. El canon de la novela negra y policíaca. Tejuelo. Didáctica de la Lengua y la Literatura. Educación. 2008: (1): 58-74.

17. Fischler C. L'Homnivore. Paris: Editions Odile Jacob; 1993. 
18. Contreras J, compilador. Alimentación y Cultura. Necesidades, gustos y costumbres. Barcelona: Universitat de Barcelona; 2002.

19. Torres-Pancalvo P.A. El cine y la gastronomía. Madrid: Editorial Visión Libros; 2011.

20. Hidalgo-Mari T, Segarra-Saavedra J, Rodríguez-Monteagudo E. La gastronomía como temática recurrente en el cine: un recorrido cualitativo por las películas más representativas con contenido culinario. Actas. VI Congreso Internacional Latina de Comunicación Social. Tenerife: Universidad de La Laguna; 2014

21. Dos Ramos M. ¿Cueces o enriqueces? Género, cocina y representación en el cine contemporáneo. Dossiers Feministes 2013. 17:81-97.
22. Barthes R. Pour une psicho-sociologie de I'alimentation contemporaine. Annales. 1961. 16: 977-986.

23. Contreras J, Gracia A M. Alimentación y Cultura. Perspectivas Antropológicas. Barcelona: Ariel; 2010.

24. Ross M. Los siete pasos de la danza del comer. Cultura, género e identidades. San José: Editorial Universitaria de Costa Rica; 2009.

25. Braudel F. El Mediterráneo y el mundo mediterráneo en la época de Felipe II. Barcelona: FCE; 1976.

26. Urroz Arancibia JJ. La gastronomía en los medios de comunicación. Una visión crítica. Revista Ábaco. El impacto mediático de la gastronomía 2008. 57: 19-34.

Rafael Cartay. Economista (Universidad Central de Venezuela). Maes-
tría en Economía Agrícola (Colegio de Postgraduados Chapingo,
IICA-OEA, Turrialba, Costa Rica). Dr. Tercer Ciclo (EPHE-Université
Paris I-Sorbonne, París, Francia).
Universidad de los Andes, Venezuela, Centro de Investigaciones
Agroalimentarias “Edgar Abreu Olivo" (CIAAL-EAO). Docente invi-
tado Universidad Técnica de Manabí, Portoviejo, Manabí, Ecuador.

